\title{
The relationship between frequency of carbohydrates intake and dental caries: a cross-sectional study in Italian teenagers $\dagger$
}

\author{
Davide Arcella ${ }^{1}$, Livia Ottolenghi ${ }^{2}$, Antonella Polimeni ${ }^{2}$ and Catherine Leclercq ${ }^{1, *}$ \\ ${ }^{1}$ National Institute for Food and Nutrition Research (INRAN), Via Ardeatina 546, I-00178 Rome, Italy: \\ ${ }^{2}$ Department of Paediatric Dentistry, University 'La Sapienza', Rome, Italy
}

Submitted 4 September 2001: Accepted 14 November 2001

\begin{abstract}
Objective: To propose an approach for investigation of the relationship between the frequency of carbohydrates intake and dental caries in real-life conditions.

Design: The frequency of separate eating events (with the exclusion of lunch and dinner) and their sugars and starch content were assessed on the basis of diaries collected for seven consecutive days. The total number of decayed, missing and filled teeth (DMFT) was assessed through clinical examination. The interrelation between DMFT and frequency of eating events was analysed through Pearson correlation coefficient and stepwise forward linear regression analysis.

Setting: Italy.

Subjects: One hundred and ninety-three students (males and females, mean age 16 years) of a secondary school.

Results: Individual DMFT varied between 0 (24\% of subjects) and 12, with a mean of 3. Once lunch and dinner were excluded, the mean number of separate eating events was 2.9. A statistically significant relationship was found between DMFT and eating frequency thus defined. Correlations were calculated considering only eating events containing a proportion of sugars or starch higher than different cut-off levels. In a stepwise multiple regression model for DMFT, the frequency of 'high sugars and high starch events' accounted for 8\% of the DMFT variance. Overall frequency of separate eating events defined according to their content of both sugars and starch accounted for $18 \%$ of the DMFT variance. A stronger correlation was found among males only. Conclusions: The precise characterisation of eating events in terms of their sugars and starch content allows us to explain a consistent percentage of the variability in DMFT.

Eating events Keywords Caries Sugars Starch Stepwise linear regression analysis
\end{abstract}

Dental caries has a multifactorial aetiology including diet, genetics, fluoride exposure and behaviour ${ }^{1}$. Dental decay depends directly on plaque bacteria that metabolise fermentable carbohydrates: the acids that are thus formed lead to the demineralisation of teeth. Each meal containing carbohydrates leads to an acid attack. The demineralisation process depends not only on the daily number of acid attacks but also on their duration and intensity, which are related to the quantity, physical status and composition of food. The frequency and typology of snacks consumed out of conventional mealtimes, and in particular the content of sugars and starch, are thus important factors. A recent extensive review of the relationship between diet and dental diseases confirms the importance of the frequency of consumption of sugars in the aetiology of caries: the intake of extrinsic sugars beyond four times a

tThis paper was awarded as the 'best original work presented by an under-35 scientist' during the Congress of the Italian Society of Human Nutrition (Naples, March 2000). day leads to an increased risk of dental caries ${ }^{2}$. Starches, if added with sugar, are also highly cariogenic ${ }^{3}$. However, in numerous epidemiological studies, the correlation observed between oral health and frequency of sugars intake was very low or null - i.e. the frequency of intake was found to explain only a small proportion of caries variance. This was observed in both longitudinal ${ }^{4,5}$ and cross-sectional $^{6}$ studies. The low associations observed may in part be due to methodological problems in the definition and characterisation of eating events in observational studies.

The aim of this study was to propose an approach for investigation of the relationship between the frequency of carbohydrates intake and dental caries in real-life conditions.

\section{Methods}

The present study was performed within the framework of a multi-centre study co-ordinated by the Institute of 
European Food Studies (IEFS). The core part of the study, common to the five countries involved, was a food survey aimed at assessing the effect of survey duration on the estimation of food chemical intake $\mathrm{e}^{7,8}$. The protocol of the Italian centre also included an ad boc codification of sugar-free products aimed at assessing the pattern of use of sugar-free chewing gums with a view to caries protection ${ }^{9}$ and the intake exposure to intense sweeteners ${ }^{10}$. Moreover, a clinical examination of dental health was performed for the purpose of investigations referred to in the present paper. Some preliminary results were presented at International Epidemiological Association meeting in $1999^{11}$ and the Italian Human Nutrition Society meeting in $2000^{12}$.

\section{Study population}

All the students of 11 classes (second, third and fourth year) of a secondary school of Rome, Italy, were invited to participate in the study to constitute a convenience sample. All students agreed to participate. Of the 241 boys and girls who were invited to participate, 48 subjects were excluded for different reasons: absent from school during the study $(n=1)$, did not complete all diaries $(n=6)$, were found to be unreliable in reporting intakes by the interviewers $(n=15)$, were absent at the medical examination $(n=25)$ or presented serious teeth disease $(n=1)$. Participants were mostly female $(74 \%)$. One hundred and sixty-seven subjects (87\%) were aged 15-17 years, only one subject was 13 years old and one 19 years old; mean age (standard deviation (SD)) was 16 (1.1) years in males and 16 (1.0) years in females.

\section{Caries assessment}

Dental examinations were performed by two experienced dentists following World Health Organization (WHO) recommendations for oral health surveys ${ }^{13}$. Both enamel caries and dentine caries were registered but no radiographs were taken. From this information, the total number of decayed, missing or filled permanent teeth (DMFT index) was calculated for each subject.

\section{Dietary assessment}

The food survey was conducted from September 1996 to December 1996. Subjects were asked to list sequentially in diaries all foods and beverages ingested during 14 consecutive days with the exact time of consumption. The diaries were structured so that each eating event was registered as breakfast, lunch, dinner or snack (midmorning, mid-afternoon, evening). The layout of the diaries enabled details of every eating occasion to be recorded: description of food, quantity consumed, unit of measurement, added seasonings, main ingredients of home-made recipes and the precise name of manufactured foods. Previous analysis showed that the quality of the present consumption data decreased after seven days: the average number of snacks was $21 \%$ lower in the second week with respect to the first, probably due to some degree of tiredness of subjects ${ }^{7}$. As performed by other authors ${ }^{14}$, only the first week of the survey was therefore considered in the present investigation.

Students were instructed on how to express quantities with household units of measurement. Manufactured products were recorded with the number (or fraction) of single units consumed. Students were asked to fill in the diaries after each eating occasion if possible, to maintain their usual eating habits and to report if they followed a particular diet during the survey. Diaries were controlled three times a week through individual interviews. The interviewers went through the previous day's entries with each student to check food description and to identify possible omissions such as between-meal eating occasions (beverages, candies, chewing gums, snacks, etc.) and added sweeteners.

For the analysis three different databanks were used.

1. The 'units databank' associated to the food portions and measures of each single food/recipe to their weight in grams. Standard Italian food portion sizes (Italian Society of Human Nutrition 1996) were used for non-manufactured foods.

2. The 'recipes databank' ${ }^{15}$ transformed the weight of mixed dishes into the weight of raw single foods (ingredients).

3. The Italian National Tables of Food Composition ${ }^{16}$ were used to calculate the sugars (mono- and disaccharides, expressed as monosaccharides) and starch (polysaccharides) content of each single eating occasion.

These databanks were updated through insertion of new recipes and manufactured products consumed by students during the survey. Subjects were asked to keep the packaging of new or unusual foods consumed during the survey so that the exact weight/volume and the content of sugars and starch could be retrieved from the labels.

Rigorous quality control of the procedure of codification and data entry were performed, as already reported elsewhere $^{10}$.

An eating event was defined as the sum of food or beverage items, with the exclusion of drinks of only water, consumed simultaneously (e.g. tea plus sugar plus biscuits, all registered at 5:05 p.m.). Only breakfast and snacks were considered, lunch and dinner were never considered. Whether an eating event was breakfast, lunch, dinner or a snack was determined by the participant, not by time of day. An eating event was contemplated if the time from the previous intake was higher than a defined interval. Different time intervals were tested, from none (e.g. taking into account all eating events) to $60 \mathrm{~min}$, in order to optimise the definition of 'separate eating events'. The overall mean concentration of sugars and starch actually present in the mouth was calculated for every 
eating event as a weighed mean of the concentration of all foods and beverages consumed. The number of separate eating events containing a proportion of sugars/starch higher than a cut-off level was calculated for each subject.

Finally, each eating occasion was assigned to one of nine groups according to the simultaneous concentration of sugars (low, average, high) and starch (low, average, high). The cut-off levels used to define these groups derive from a correlation analysis (see Statistical analysis section) and are reported in the Results section. The overall frequency of separate eating events was calculated within each group.

As usually performed in nutritional surveys, EI/BMR ratio (ratio of total energy intake to basal metabolic rate) was used as an indicator of underreporting ${ }^{17}$.

\section{Statistical analysis}

Raw data files were incorporated into a SAS ${ }^{18}$ database for subsequent statistical analysis. Means, frequency distributions and standard deviations were determined in the descriptive analysis of the variables. Differences were tested using the $t$-test or $F$-test. The Pearson correlation coefficient was used as a measure of association between caries and diet; for all of the values reported in the figures $P$ was less than 0.05 . The correlation between DMFT and the frequency of eating occasions containing a proportion of sugars or starch higher than a cut-off point was calculated, varying the cut-off point.

A stepwise forward linear regression analysis was performed to study the interrelations between DMFT (dependent variable) and the frequency of the nine different groups of eating events and age (independent variables). All variables that were significant at the 0.10 level were included in the model. This analysis was also performed for groups according to sex.

\section{Results}

Individual DMFT varied between 0 (24\% of subjects) and 12. Mean DMFT did not differ significantly between males and females $\left(t_{191}=-1.18, P=0.24\right)$, mean DMFT was 2.6 $(\mathrm{SD}=2.3 ; n=51)$ for the former and $3.2(\mathrm{SD}=2.9$; $n=142$ ) for the latter. Mean DMFT increased from 2.5 $(\mathrm{SD}=2.5 ; n=64)$ in age group $13-15$ years to 3.1 ( $\mathrm{SD}=$ $2.8 ; n=69)$ in age group 16 years and to 3.6 ( $\mathrm{SD}=2.9$; $n=60)$ in age group $17-19$ years; also, in this case, the differences were not statistically significant $\left(F_{2,190}=2.60\right.$, $P=0.0772)$. Mean EI/BMR ratio in non-slimming subjects was 1.69 in males versus 1.55 in females, suggesting that no gross underreporting occurred. No subject declared to be diabetic and 14 subjects (all females) declared to be on a slimming diet during the survey. In all but one of these females, EI/BMR ratio was in fact under 1.2.

Correlations between DMFT and the frequency of eating occasions containing a proportion of sugars or starch higher than defined cut-off levels are reported in Figs 1 and 2, respectively, with cut-off increasing from $0 \%$ (i.e. no cut-off) to 50\%. Data are shown for only two time intervals: none and $45 \mathrm{~min}$. At all sugar cut-off levels and most starch ones, the correlation was higher when only eating events more than $45 \mathrm{~min}$ distant from the previous intake were considered. With the other time intervals tested, the correlation coefficients were slightly lower (data not shown). When no cut-off levels for sugar and starch were applied the correlation coefficients were 0.30 and 0.31 , respectively, for no cutoff for time interval and for $45 \mathrm{~min}$.

In the case of sugars (Fig. 1), a U-shaped curve relates the correlation with the cut-off when this varies from $0 \%$ to $10 \%$. The highest correlation (0.32) was obtained with the $10 \%$ cut-off, i.e. by correlating DMFT with the number of

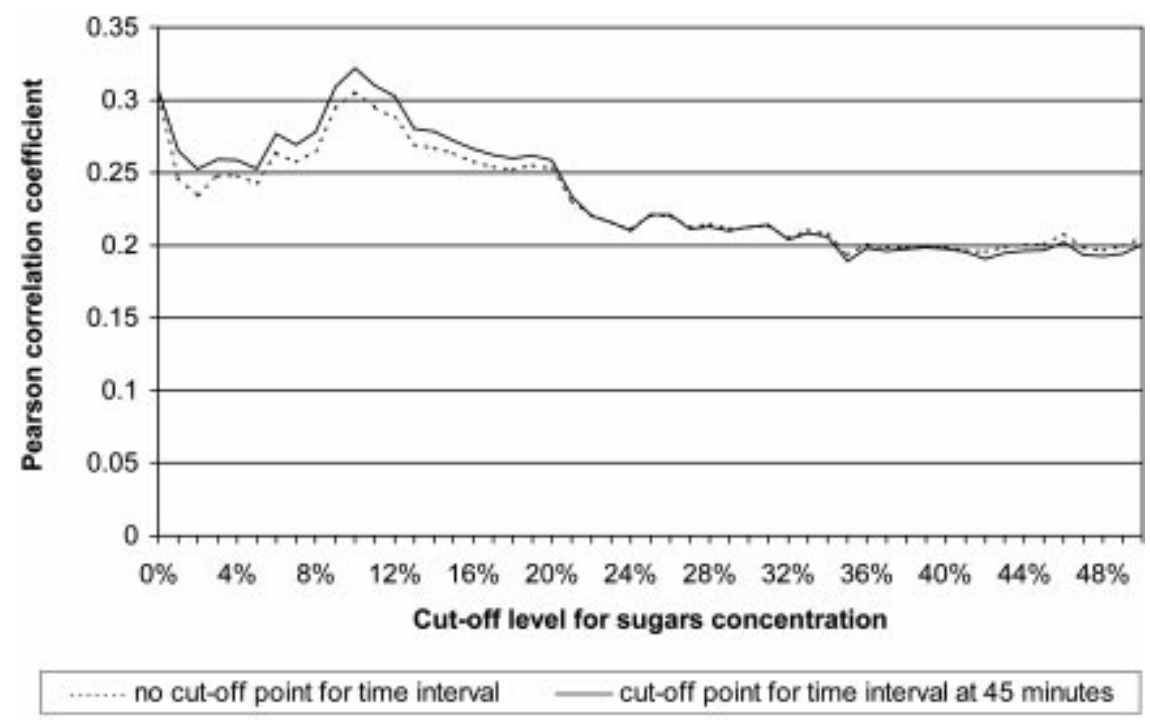

Fig. 1 Pearson correlation coefficient between DMFT and frequency of eating occasions containing a proportion of sugars higher than a cut-off level 


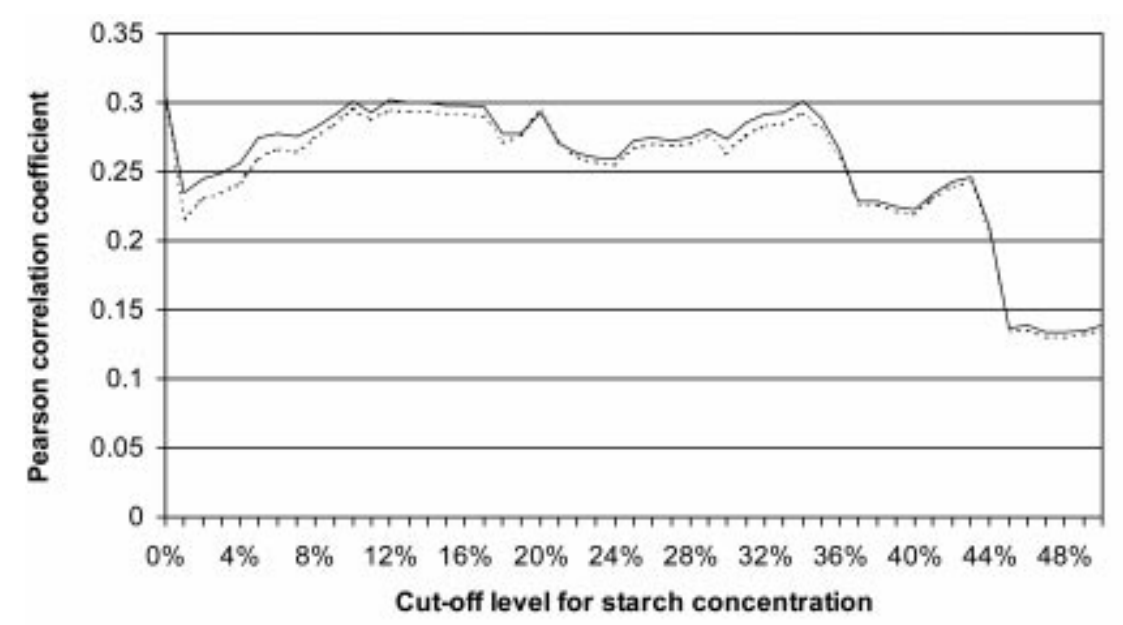

no cut-off point for time interval _ cut-off point for time interval at 45 minutes

Fig. 2 Pearson correlation coefficient between DMFT and frequency of eating occasions containing a proportion of starch higher than a cut-off level

eating events containing at least $10 \%$ of sugars. The correlation decreased significantly when the cut-off was set above $20 \%$.

The correlation between DMFT and the number of eating occasions was more homogeneous when varying the cut-off level for starch (Fig. 2). The highest value (0.3) was found when cut-off was set at 0\% (no cut-off), 12\% and $34 \%$, with a U-shaped curve from 0 to $12 \%$ and a quick drop above $34 \%$.

Concentration levels at which the shapes of the two curves mentioned above were shown to change were used as cut-off levels to classify eating events: low sugars (under 10\%), average sugars (between 10\% and 20\%) and high sugars (over 20\%); low starch (under 12\%), average starch (between 12\% and 34\%) and high starch (over 34\%). Eating occasions were therefore assigned to one of nine groups according to the simultaneous concentration of sugars (low, average, high) and starch (low, average, high). Moreover, a separate eating event was considered if the time from the previous intake was greater than $45 \mathrm{~min}$.

In a stepwise multiple regression model for DMFT (Table 1), the frequency of separate eating events of four groups of eating events was included in the model, with the variable 'high sugars and high starch events' accounting for $8 \%$ of variance and the three other variables for 3 to $4 \%$. Overall these four variables accounted for $18 \%$ of the DMFT variance. The fifth variable entered in the model was age, explaining almost $3 \%$ of the DMFT variance.

The presence of different food groups, defined according to their potential cariogenicity, in each of the nine categories of eating events is described in Table 2. The 'high sugars and high starch events' most often comprised sweetened baked goods (93\%). 'Low sugars and high starch events' were mainly sandwiches, pizza and snacks (94\%), whereas 84\% of 'high sugars and low starch events' contained confectionery products. The 'average sugar and average starch events' are more difficult to define; the majority of these (84\%) contained sweetened baked goods but the presence of table sugar and sweeteners, milk and sweet dairy products, soft drinks and fruit juice and confectionery was also noteworthy.

Among males, four independent variables were included in the model accounting for almost 54\% of the DMFT variance (Table 3). Age did not enter in the model. For females, the four independent variables entered in the model explained $17 \%$ of the DMFT variance (Table 4). Age (3.5\%) was the second variable entered in the model.

Table 1 Summary of stepwise procedure for DMFT (dependent variable) and number of separate eating events grouped according to sugars and starch concentration (independent variable) in the whole sample

\begin{tabular}{llcrrc}
\hline \multicolumn{1}{c}{ Independent variable } & Partial $R^{2}$ & Cumulative $R^{2}$ & $F$-value & Probability $F$ \\
\hline 1 & High sugars and high starch events & 0.0791 & 0.0791 & 16.4039 & 0.0001 \\
2 & High sugars and low starch events & 0.0375 & 0.1166 & 8.0688 & 0.0050 \\
3 & Average sugars and average starch events & 0.0345 & 0.1511 & 7.6837 & 0.0061 \\
4 & Low sugars and high starch events & 0.0284 & 0.1795 & 6.4978 & 0.0116 \\
5 & Age & 0.0255 & 0.2049 & 5.9921 & 0.0153 \\
\hline
\end{tabular}

All variables left in the model are significant at the 0.10 level.

The other variables did not meet the 0.10 significance level for entry into the model.

$n=193$. 
As can be seen from Table 5, eating events were more frequent in females than in males: 3.11 versus 2.45 $\left(F_{1,191}=20.22, P=0.0001\right)$. In particular, females had a significantly higher intakes of 'high sugars and low starch events' $\left(F_{1,191}=14.48, P=0.0002\right)$, 'low sugars and low starch events' $\left(F_{1,191}=5.82, P=0.0167\right)$ and 'average sugars and high starch events' $\left(F_{1,191}=6.58, P=0.0111\right)$.

\section{Discussion}

Since the purpose of this study was to identify potentially cariogenic eating patterns, special attention was given to the characterisation of eating events - defined as the sum of food or beverage items consumed at the same time and to the time between these events, in order to obtain a detailed picture of the number and type of acid attacks for each individual within the survey period. A specific aspect of our study is the attempt to establish cut off-points for both the time interval between eating events and the concentration of sugars and/or starch, in order to improve the predictive capacity of the frequency of eating events.

Many other cross-sectional studies have investigated the relationship between DMFT and frequency of eating events. The cariogenicity of an eating event is usually assessed on the basis of the type of foods consumed as categorised according to their physical characteristics and/or rough carbohydrate content ${ }^{3,19,20}$, leading to a low percentage of DMFT variance explained by either sugars or starch events. According to some authors, the relative cariogenicity of a food is not correlated with the amount of carbohydrates it contains and it is not possible to estimate the minimal cariogenic concentration of sugars in foods, since this threshold varies with too many different factors $^{21}$. The results obtained in the present study suggest that this issue might deserve more consideration. In the present study, the concentration of sugars and starch actually present in the mouth was calculated for every eating event with the use of food composition tables. To our knowledge, this computation has been performed previously by other authors in order to characterise the patterns of sugar intake ${ }^{22}$ but was not put in relation to dental decay. In our case, the overall frequency of separate eating events defined according to their concentration with respect to cut-offs for both sugars and starch accounted for $18 \%$ of the DMFT variance. The importance of considering concurrently the presence of starch and sugars was made clear by the observation of two curves reporting the correlations between DMFT and the frequency of eating occasions according to the cut-off levels used to define sugars and starch events (Figs 1 and 2). In fact, by applying a low cut-off for sugar, the correlation obtained was lower than that obtained considering no cut-off, i.e. all eating events. This is probably due to the fact that in this case eating events with no or very low sugars were excluded and a large number of these were eating occasions with high starch (such as 
Table 3 Summary of stepwise procedure for DMFT (dependent variable) and number of separate eating events grouped according to sugars and starch concentration (independent variable) in males

\begin{tabular}{llrrrc}
\hline & Independent variable & Partial $R^{2}$ & Cumulative $R^{2}$ & $F$-value & Probability $F$ \\
\hline 1 & Average sugars and average starch events & 0.2493 & 0.2493 & 16.2700 & 0.0002 \\
2 & Average sugars and high starch events & 0.1122 & 0.3614 & 8.4309 & 0.0056 \\
3 & Low sugars and high starch events & 0.1415 & 0.5029 & 13.3797 & 0.0006 \\
4 & High sugars and high starch events & 0.0326 & 0.5356 & 3.2320 & 0.0788 \\
\hline
\end{tabular}

All variables left in the model were significant at the 0.10 level.

No other variable met the 0.10 significance level for entry into the model. $n=51$.

pizza), which are potentially cariogenic. Similarly, by excluding eating events with very low starch, cariogenic eating events with no starch and high sugars (such as confectionery) are excluded. For this reason, eating events were grouped according to their content of both sugars and starch.

Only a few studies have given attention to the definition of separate eating events (considering a minimum time interval between different intake events). Some authors considered that eating events were separate if the time of consumption was at least $30 \mathrm{~min}$ after the previous intake if this was a meal, or at least $15 \mathrm{~min}$ if it was classified as a snack $^{19,20}$. In our sample, the optimal time interval cut-off was found to be $45 \mathrm{~min}$.

In most similar studies, main meals (breakfast, lunch and dinner) were not considered in the computation of eating events since they are taken by all subjects and do not introduce variability in the number of acid attacks. Our choice to consider both breakfast and snacks, excluding only lunch and dinner, is related to a specificity of current Italian eating patterns: breakfast is not taken by the whole population. In our sample $20 \%$ of teenagers did not have breakfast regularly; the correlation observed between eating events and DMFT was lower if breakfast was not considered (data not shown).

A shortcoming common to all cross-sectional studies regarding this topic is the use of current eating patterns as a proxy for earlier ones, which are those actually related with observed caries levels.

A specific drawback of the present data collection is that drinks of water were not registered; in some cases, the concentration of sugars and starch in the oral cavity may be significantly reduced by the simultaneous consumption of water. A further specification of eating events could have been obtained through registration of the food sequence within each eating event, since it is reasonable to assume that the cariogenic effect of a food rich in sugar and/or starch is higher if this is the last item consumed within the eating occasion. Moreover, the individual oral retentiveness of foods containing sugars and/or starch, an important factor associated with caries incidence, was not taken into account ${ }^{20}$. If these aspects had been considered, the frequency of eating events containing sugars and/or starch might have explained an even higher percentage of DMFT variance.

Apart from sugars and starch, many other factors are known to be involved in the aetiology of dental decay: genetics, oral hygiene, topical use of fluoride through toothpaste, intake of both fluoride and other nutrients. These factors explain the percentage of variability not explained by the frequency of eating events. It cannot be excluded that some of these may have acted as confounding factors, leading to a spurious correlation between frequency of eating events and DMFT. However, it is unlikely for example that genetic susceptibility to dental decay is related to the frequency of sugars and/or starch events. Moreover, the relationship observed is biologically plausible and consistent with that of experimental studies.

The fact that the sample was unbalanced for sex is due to our choice of selecting a convenience sample (11 full classes of a unique school). This choice was made necessary by the large amount of resources needed to perform dental examinations and to collect food consumption data at such a high level of detail. However, the school chosen was in a medium class neighbourhood,

Table 4 Summary of stepwise procedure for DMFT (dependent variable) and number of separate eating events grouped according to sugars and starch concentration (independent variable) in females

\begin{tabular}{llccrc}
\hline \multicolumn{1}{c}{ Independent variable } & Partial $R^{2}$ & Cumulative $R^{2}$ & $F$-value & Probability $F$ \\
\hline 1 & High sugars and high starch events & 0.0677 & 0.0677 & 10.1633 & 0.0018 \\
2 & Age & 0.0348 & 0.1025 & 5.3871 & 0.0217 \\
3 & High sugars and low starch events & 0.0419 & 0.1444 & 6.7650 & 0.0103 \\
4 & Low sugars and high starch events & 0.0275 & 0.1719 & 4.5538 & 0.0346 \\
\hline
\end{tabular}

All variables left in the model were significant at the 0.10 level.

No other variable met the 0.10 significance level for entry into the model.

$n=142$. 
Table 5 Average daily number of eating events with the exclusion of lunch and dinner, by sex

\begin{tabular}{lccc}
\hline & \multicolumn{3}{c}{ Average daily intake } \\
\cline { 2 - 4 } Eating event & Among males & Among females & In the whole sample \\
\hline Low sugars and low starch events & 0.70 & 0.91 & 0.86 \\
Low sugars and average starch events & 0.37 & 0.30 & 0.32 \\
Low sugars and high starch events & 0.21 & 0.30 & 0.27 \\
Average sugars and low starch events & 0.50 & 0.54 & 0.53 \\
Average sugars and average starch events & 0.15 & 0.10 & 0.11 \\
Average sugars and high starch events & 0.02 & 0.08 & 0.06 \\
High sugars and low starch events & 0.33 & 0.63 & 0.55 \\
High sugars and average starch events & 0.14 & 0.20 & 0.18 \\
High sugars and high starch events & 0.03 & 0.06 & 0.05 \\
Total events & 2.45 & 3.11 & 2.93 \\
\hline
\end{tabular}

all of the students contacted participated in the study and the compliance to the study protocol was high; only $20 \%$ of subjects were excluded for different reasons. This makes us fairly confident in relation to the absence of a selection bias and allows us to consider that the results represent the situation of fairly typical Italian students of medium social class.

The stronger correlation found among males cannot be unambiguously explained. The different dietary pattern observed between the two sex groups could be responsible for the higher percentage of caries variance explained by the frequency of eating events in males. Even though teenagers did not differ for the self-reported frequency of teeth brushing (data not shown), there is the possibility that the higher association between eating occasions frequency and DMFT among males could be related to a poorer oral hygiene in this group. It is in fact noteworthy that the association between sugars and dental decay is stronger in the case of poorer oral hygiene ${ }^{21}$.

In conclusion, this study suggests that further characterisation of eating events in real-life conditions deserves more attention - in particular in relation to their sugars and starch content. The approach proposed in this paper could be an efficient tool for an in-depth study of the relationship between pattern of carbohydrates consumption and dental caries in real-life conditions.

\section{Acknowledgements}

The authors are most thankful to Dario Berardi, Roberto Cerreto, Lucrezia Leomporra, Francesca Morichetti and Maria Rita Sorbillo for their help in the study.

\section{References}

1 Konig KG, Navia JM. Nutritional role of sugars in oral health. Am.J. Clin. Nutr. 1995; 62(Suppl. 1): 275S-82S.

2 Sheiham A. Dietary effects on dental diseases. Public Health Nutr. 2001; 4(2B): 569-91.

3 García-Closas R, García-Closas M, Serra-Majem L. A crosssectional study of dental caries, intake of confectionery and foods rich in starch and sugars, and salivary counts of
Streptococcus mutans in children in Spain. Am.J. Clin. Nutr. 1997; 66(5): 1257-63.

4 Rugg-Gunn AJ, Hackett AF, Appleton DR, Jenkins GN, Eastoe JE. Relationship between dietary habits and caries increment assessed over two years in 405 English adolescent school children. Arch. Oral Biol. 1984; 29(12): 983-92.

5 Burt BA, Eklund SA, Morgan KJ, Larkin FE, Guire KE, Brown LO, et al. The effects of sugars intake and frequency of ingestion on dental caries increment in a three-year longitudinal study. J. Dental Res. 1988; 67(11): 1422-9.

6 Woodward M, Walker AR. Sugar consumption and dental caries: evidence from 90 countries. Br. Dental J. 1994; 176(8): 297-302

7 Institute of European Food Studies (IEFS). The Effect of Survey Duration on the Estimation of Food Chemical Intakes. Report No. 3. Dublin: IEFS, 1998.

8 Lambe J, Kearney J, Leclercq C, Zunft HF, De Henauw S, Lamberg-Allardt CJ, et al. The influence of survey duration on estimates of food intakes and its relevance for public health nutrition and food safety issues. Eur. J. Clin. Nutr. 2000; 54(2): 166-73.

9 Leclercq C, Leomporra L, Berardi D, Sorbillo MR, Ottolenghi L, Polimeni A. An analysis of the consumption pattern of sugar free chewing gums in a sample of Italian teenagers with a view of caries protection. In: Milano prevenzione Nutrizione Genetica Ambiente, dal bambino all'adulto. XXX Riunione Generale Società Italiana di Nutrizione Umana, Milan, Italy, 26-29 November, 1998 Milan: CSH srl, 1998.

10 Leclercq C, Berardi D, Sorbillo MR, Lambe J. Intake of saccharin, aspartame, acesulfame $\mathrm{K}$ and cyclamate in Italian teenagers: present levels and projections. Food Additives Contam. 1999; 16(3): 99-109.

11 Leclercq C, Cerreto R, Berardi D, Arcella D, Polimeni A, Ottolenghi L. Association between dental caries and eating events in a sample of Italian teenagers. In: Epidemiology for Sustainable Health. Proceedings of the XV International Scientific Meeting of the International Epidemiological Association, Florence, Italy 31 August-4 September, 1999 Florence: IEA, 1999.

12 Arcella D, Ottolenghi L, Polimeni A, Berardi D, Leclercq C. Individuazione degli eventi cariogeni attraverso l'analisi della correlazione tra carie dentale e occasioni di consumo. Giornale Italiano di Nutrizione Clinica e Metabolismo 2000; 9: 48.

13 World Health Organization (WHO). Oral Health Surveys Basic Methods. Geneva: WHO, 1987.

14 Akpata ES, al-Shammery AR, Saeed HI. Dental caries, sugar consumption and restorative dental care in 12-13-year-old children in Riyadh, Saudi Arabia. Community Dentist. Oral Epidemiol. 1992; 20(6): 343-6.

15 National Institute for Food and Nutrition Research. Recipes 
database. Microdata files, National Institute for Food and Nutrition Research, Rome, 1999.

16 Carnovale E, Marletta L. Tabelle di Composizione degli Alimenti. Rome: National Institute of Nutrition, 1997.

17 World Health Organization (WHO). Energy and Protein Requirements. Report of a Joint FAO/WHO/UNU Expert Consultation. WHO Technical Report Series No. 724. Geneva: WHO, 1985.

18 SAS Institute, Inc. SAS/STAT User's Guide, Version 6.12. Cary, NC: SAS Institute, Inc., 1989.

19 Beighton D, Adamson A, Rugg-Gunn A. Associations between dietary intake, dental caries experience and salivary bacterial levels in 12-year-old English schoolchildren. Arch. Oral Biol. 1996; 41(3): 271-80.

20 Papas AS, Joshi A, Palmer CA, Giunta JL, Dwyer JT. Relationship of diet to root caries. Am. J. Clin. Nutr. 1995; 61(2): 423S-9S.

21 Kandelman D. Sugar, alternative sweeteners and meal frequency in relation to caries prevention: new perspectives. Br. J. Nutr. 1997; 77(Suppl. 1): S121-8.

22 Hackett AF, Rugg-Gunn AJ, Appleton DR, Allinson M, Eastoe JE. Sugars-eating habits of 405 11- to 14-year-old English children. Br. J. Nutr. 1984; 51(3): 347-56. 